Journal for ImmunoTherapy of Cancer

\title{
High-dimensional analysis of the adenosine pathway in high-grade serous ovarian cancer
}

\author{
Yacine Bareche, ${ }^{1}$ Sandra Pommey, ${ }^{2}$ Mayra Carneiro (D) , ${ }^{3}$ Laurence Buisseret, ${ }^{4}$ \\ Isabelle Cousineau, ${ }^{2}$ Pamela Thebault, ${ }^{2}$ Pavel Chrobak, ${ }^{2}$ Laudine Communal, ${ }^{2}$ \\ David Allard, ${ }^{1}$ Simon C Robson, ${ }^{5}$ Anne-Marie Mes-Masson, ${ }^{3}$ Diane Provencher, ${ }^{2}$ \\ Rejean Lapointe, ${ }^{3}$ John Stagg (i) ${ }^{1}$
}

To cite: Bareche Y, Pommey S, Carneiro M, et al. Highdimensional analysis of the adenosine pathway in highgrade serous ovarian cancer. Journal for ImmunoTherapy of Cancer 2021;9:e001965. doi:10.1136/jitc-2020-001965

- Prepublication history and additional material is published online only. To view please visit the journal online (http://dx.doi. org/10.1136/jitc-2020-001965).

$\mathrm{YB}, \mathrm{SP}$ and $\mathrm{MC}$ contributed equally.

$\mathrm{RL}$ and JS are joint senior authors.

Accepted 22 February 2021

Check for updates

(C) Author(s) (or their employer(s)) 2021. Re-use permitted under CC BY-NC. No commercial re-use. See rights and permissions. Published by BMJ.

For numbered affiliations see end of article.

Correspondence to

Dr John Stagg;

john.stagg@umontreal.ca

\section{ABSTRACT}

Background Hydrolysis of extracellular ATP to adenosine (eAD0) is an important immune checkpoint in cancer immunology. We here investigated the impact of the eADO pathway in high-grade serous ovarian cancer (HGSC) using multiparametric platforms.

Methods We performed a transcriptomic meta-analysis of eAD0-producing CD39 and CD73, an eAD0 signaling gene signature, immune gene signatures and clinical outcomes in approximately 1200 patients with HGSC. Protein expression, localization and prognostic impact of CD39, CD73 and CD8 were then performed on approximately 1000 cases on tissue microarray, and tumor-infiltrating lymphocytes (TILS) were analyzed by flow cytometry and single-cell RNA sequencing on a subset of patients.

Results Concomitant CD39 and CD73 gene expression, as well as high levels of an eAD0 gene signature, were associated with worse prognosis in patients with HGSC, notably in the immunoregulatory molecular subtype, characterized by an immune-active microenvironment. CD39 was further associated with primary chemorefractory and chemoresistant human HGSC and platinum-based chemotherapy of murine HGSC was significantly more effective in CD39-deficient mice. At protein level, CD39 and CD73 were predominantly expressed by cancer-associated fibroblasts, and CD39 was expressed on severely exhausted, clonally expanded and putative tissue-resident memory TILs.

Conclusions Our study revealed the clinical, immunological, subtype-specific impacts of eADO signaling in HGSC, unveiled the chemoprotective effect of CD39 and supports the evaluation of eADO-targeting agents in patients with ovarian cancer.

\section{INTRODUCTION}

Increased lymphocytic infiltration in highgrade serous ovarian cancer (HGSC) is associated with improved overall survival (OS), supporting the rationale to develop immunotherapeutic strategies. ${ }^{1-3}$ However, immune checkpoint inhibitors targeting programmed cell death protein 1 (PD-1) or programmed death-ligand 1 (PD-L1) have yet to show meaningful clinical impact in HGSC as single agents, with overall response rates ranging from $8 \%$ to $15 \% .{ }^{4}$ Reported synergistic activities with chemotherapy, poly(ADP-ribose) polymerase inhibitors, ${ }^{5}$ antiangiogenic agents, radiation therapy and other immunotherapies ${ }^{6}$ raise new hopes for combinatorial approaches.

Among emerging cancer immunotherapies are inhibitors of extracellular adenosine (eADO) signaling, a pathway mainly regulated by the ectonucleotidases CD39 and CD73 and the $\mathrm{G}$ protein-coupled receptors A2A and A2B. ${ }^{7}$ CD39 hydrolyses extracellular ATP into AMP, while CD73 hydrolyses extracellular AMP into eADO. Targeted blockade of CD39, CD73, A2A and/or A2B receptors enhances anti-tumor immunity in preclinical cancer models. ${ }^{78}$ Whether targeting eADO signaling represents a therapeutic avenue for HGSC still remains to be determined.

Previous studies, including from our group, reported that CD73 gene and protein expression in HGSC are significantly associated with worse overall and progression-free survival. ${ }^{9}$ In the ID8 mouse model of ovarian cancer, inhibition of CD73 or A2A receptor enhanced antitumor immunity and suppressed tumor growth. ${ }^{1011}$ CD73 has also been associated with ovarian cancer stemness ${ }^{12}$ and platinum resistance. ${ }^{13}$ The clinical and biological impact of CD39 in ovarian cancer, on the other hand, is largely uncharacterized. CD39 has been found expressed by ovarian tumor-associated macrophages, ${ }^{14} 15$ T regulatory cells (Tregs) ${ }^{16}$ and terminally exhausted $\mathrm{CD}^{+}$tumor-infiltrating lymphocytes (TILs). Notably, CD39 expression on TILs has been associated with tumor antigen reactivity. ${ }^{17}$

We here performed gene expression meta-analysis of the eADO pathway, protein expression analysis of CD39 and CD73 and flow cytometry analysis and single-cell RNA 
sequencing (scRNAseq) of ovarian TILs to investigate the clinical impact of eADO in HGSC.

\section{METHODS}

\section{Patients and gene expression analysis}

Datasets were processed from the curated MetaGxOvarian R package. ${ }^{18}$ Analysis was restricted to datasets featuring microarray-based whole-transcriptome studies of at least 40 patients with high-grade, primary tumors of ovarian serous histology and OS data. This resulted in nine studies providing data for 1161 patients with long-term follow-up. For each dataset, each gene was scaled using the genefu R package. ${ }^{19}$ Molecular classification was assigned for each dataset separately using the ConsensusOV $\mathrm{R}$ package. ${ }^{20}$ The cytotoxic (CYT), ${ }^{21}$ ADO signature ${ }^{22}$ and interferon (IFN) signature ${ }^{23}$ have been described. The chemoresistant HGSC datase ${ }^{24}$ is hosted by the International Cancer Genome Consortium under the accession code: OV-AU. It contains raw counts from RNA-sequencing of 93 HGSC patients with linked patient's clinical and survival information. Raw counts expression data were normalized by computing $\log 2$ transcript per million method.

\section{Patients and immunofluorescence analysis}

Co-immunofluorescence (IF) was performed in a previously described cohort of 200 HGSC on tissue microarray $(\mathrm{TMA})^{9}$ and in a previously described cohort of 1000 HGSC (from the Canadian Ovarian Experimental Unified Resource) ${ }^{25}$ on TMA (online supplemental table S1). Only cases that did not receive neoadjuvant chemotherapy were included for analyses. Slides were deparaffinized, rehydrated, demasked using a citrate buffer and incubated for $30 \mathrm{~min}$ with a protein blocking solution. Primary antibodies were co-incubated on the slides overnight at $4^{\circ} \mathrm{C}$ and secondary antibodies were incubated for 2 hours at room temperature (online supplemental table S2). Slides were then mounted with 4',6-diamidino-2-phenylindole (DAPI). Sections were imaged at 20X magnification on VS-110 scanner (Olympus) and super images were imported into Visiomorph DP (Visiopharm). CK8-18 positivity was used to identify the epithelial area and where indicated $\alpha$ smooth muscle actin (SMA) positivity was used to identify the activated stromal area. Cores with epithelial or stromal areas representing less than 5\% of the total tissue area were eliminated from survival analyses. Recommendations for Tumor Marker Prognostic Studies criteria were followed. ${ }^{26}$

\section{Patients and TIL analysis}

Fresh tissue was collected from five newly diagnosed treatment-naive patients undergoing primary cytoreductive surgery for high-grade epithelial ovarian cancer at Montreal University Hospital Center (CHUM), and processed to a single-cell suspension using GentleMACS (MACS Miltenyi). Isolated CD $45^{+}$cells were cryopreserved in $90 \%$ fetal bovine serum (FBS) and 10\% dimethyl sulfoxide (DMSO) until stained with fluorescent dyeconjugated antibodies for $30 \mathrm{~min}$ at $4^{\circ} \mathrm{C}$ (online supplemental table S2). Data were acquired on a BD Symphony instrument and analyzed using FlowJo software. DownSample plugin was used to obtain equal numbers of CD4 and CD8 cells for each patient. Events from downsamples were processed together allowing sample comparison. t-distributed stochastic neighbor embedding (tSNE) map distribution was run on $\mathrm{CD}^{+} \mathrm{CD}^{+}$and $\mathrm{CD}^{+} \mathrm{CD}^{+}$cells to reduce dimensionality of the data followed by FlowSOM for clustering. Resulting subpopulations were analyzed as described.

\section{Single-cell RNA sequencing}

$\mathrm{CD} 45^{+}$cells (as above) were analyzed using 10X Genomics Chromium technology. Cells were treated with $200 \mathrm{U} / \mathrm{mL}$ of Nuclease S7 and samples with viability greater than $80 \%$ were loaded for RNA barcoding in emulsion. For gene expression library, $50 \mathrm{ng}$ of cDNA was fragmented, end-repaired, adaptor inserted and cleaned with SPRIselect 0.8X. Sample index was inserted, fragments selected and DNA quantified by BioAnalyzer. For T cell receptor (TCR) libraries, $2 \mu \mathrm{L}$ of cDNA was used for amplification of TCR region in two rounds of PCR. After target enrichment, fragment selection was performed and product quantified. A $50 \mathrm{ng}$ of PCR product was fragmented, endrepaired, adaptor inserted and cleaned. Sample index was inserted, cleaned and quantified. Libraries were sequenced on Illumina NovaSeq 6000 (Sainte-Justine Hospital, Montreal, Canada) with minimum sequencing depth of 50000 read pairs per cell for gene expression library and 5000 reads per cell for TCR libraries.

\section{scRNA-seq analysis}

Libraries were processed using Cell Ranger (10X Genomics). From five patients, a total of 173907569 transcripts were obtained in 33670 cells. From 18658 recovered genes, an average of 1580 genes was detected per cell. Filtered contig annotation was used for T cell clonotype analysis. Analysis was performed on Seurat package. ${ }^{27}$ Cells with at least 200 features and features detected in at least three cells were included. Cell filtering comprised exclusion of dead cells and tumor cells. Following the Seurat pipeline, normalization was done by scTranform method, including regression of mitochondrial genes. For data visualization, first dimension reduction was executed using RunPCA and RunUMAP, following by clustering using FindNeighbors and FindClusters. Clusters were characterized by differentially expressed gene (DEG) analysis using the test model-based analysis of single-cell transcriptomics. ${ }^{28}$ The top genes identified, as well as known markers, were used to identify cell types. For TCR analysis, the pipeline of scRepertoire was used. ${ }^{29}$ TCR diversity was calculated by Shannon and Invert Simpson indexes.

\section{Animal studies}

All experiments were approved by the CHUM Institutional Animal Ethics Committee. Mouse HGS2 cells were 
obtained from Dr Frances Balkwill (Cancer Research UK) and were previously described. ${ }^{30}$ Syngeneic HGS2 cells $\left(10^{7}\right.$ cells $)$ were injected intraperitoneally (i.p.) in phosphate buffered saline (PBS) to WT and CD39-- mice (obtained from S.C. Robson, Harvard Medical School) and treated with carboplatin $(20 \mathrm{mg} / \mathrm{kg}$ i.p. $)$ once per week on weeks 5, 6 and 7. Mice were euthanized when signs of distress were observed or when weight loss exceeded 20\%. In an independent experiment, WT and CD39 ${ }^{-1-}$ mice were injected with $2 \times 10^{6}$ HGS2 cells s.c. and single cell suspensions were analyzed by flow cytometry (BD LSRFortessa) at day 12 for expression of Thy1.2 (clone 53-2.1; BV786; BD), CD4 (clone RM4-5; BUV737; BD), Foxp3 (clone NRRF-30; PE; eBiosciences), CD8 (clone 53-6.7; BV650; BD) and viability (eBiosciences Fixable dye; eFluor506).

\section{In vitro $\mathrm{T}$ cell activation assays}

CD39 $9^{-1-}$ mice were crossed with OT-1 transgenic mice (Jackson Lab). CD $39^{-/-}$mouse embryonic fibroblasts were transfected to express pCAGGS-mCD39 plasmid (using Nucleofector kit, Lonza) and sorted (on BD FACSAria) to $>95 \%$ purity for CD39 expression. All experiments were approval by the CHUM Animal Ethics Committee. For T cell activation assays, $10^{3}$ ovalbumin (OVA)-expressing ID8 cells per well were seeded with or without $10^{3}$ syngeneic fibroblasts onto round-bottom non-tissue culture 96 well-plates in a solution of $2.4 \mathrm{mg} / \mathrm{mL}$ methyl cellulose diluted in DMEM-10\% FBS. Spheroids were allowed to form for 24hours. OVA-specific CD8 T cells were purified by immune-magnetic negative selection (Stem cell EasySepTM, 19853) from splenocytes of OT-1 WT and OT-1 CD39 ${ }^{-1-}$ mice. The $10^{6}$ OT-1 cells per ml were stimulated for 72 hours with interleukin 2 (IL-2) $(30 \mathrm{U} / \mathrm{mL}$ ), IL-7 $(0.5 \mathrm{ng} / \mathrm{mL})$ and plate-bound anti-CD3 and antiCD28 antibodies $(5 \mu \mathrm{g} / \mathrm{mL}$ each). Activated OT-1 cells were added to the spheroids at an $\mathrm{E} / \mathrm{T}$ ratio of 10:1. After 4 hours of coculture, protein transporter inhibitors (Thermofisher, 00-4980-03) were added to the mix. Supernatants were harvested and measured for IFN- $\gamma$ by ELISA (R\&D Systems, DY485-05) following manufacturer's instructions.

\section{Statistical analyses}

Differential gene expression between two conditions was assessed by two-sided Wilcoxon Rank-Sum test and, when more than two conditions where compared, KruskalWallis test was considered.

Spearman correlation was used to evaluate the association between individual genes and gene signatures. Optimal cut-points were computed for each variable using the surv_cutpoint function form the survminer $\mathrm{R}$ package. Statistical significance of the difference in survival was computed using the Cox proportional hazard model and the log-rank test $\mathrm{p}$ value reported on KaplanMeier survival curves. ${ }^{19}$ Gene ontology (GO) enrichment analysis was performed using the goana function from the limma R package. ${ }^{31}$
A
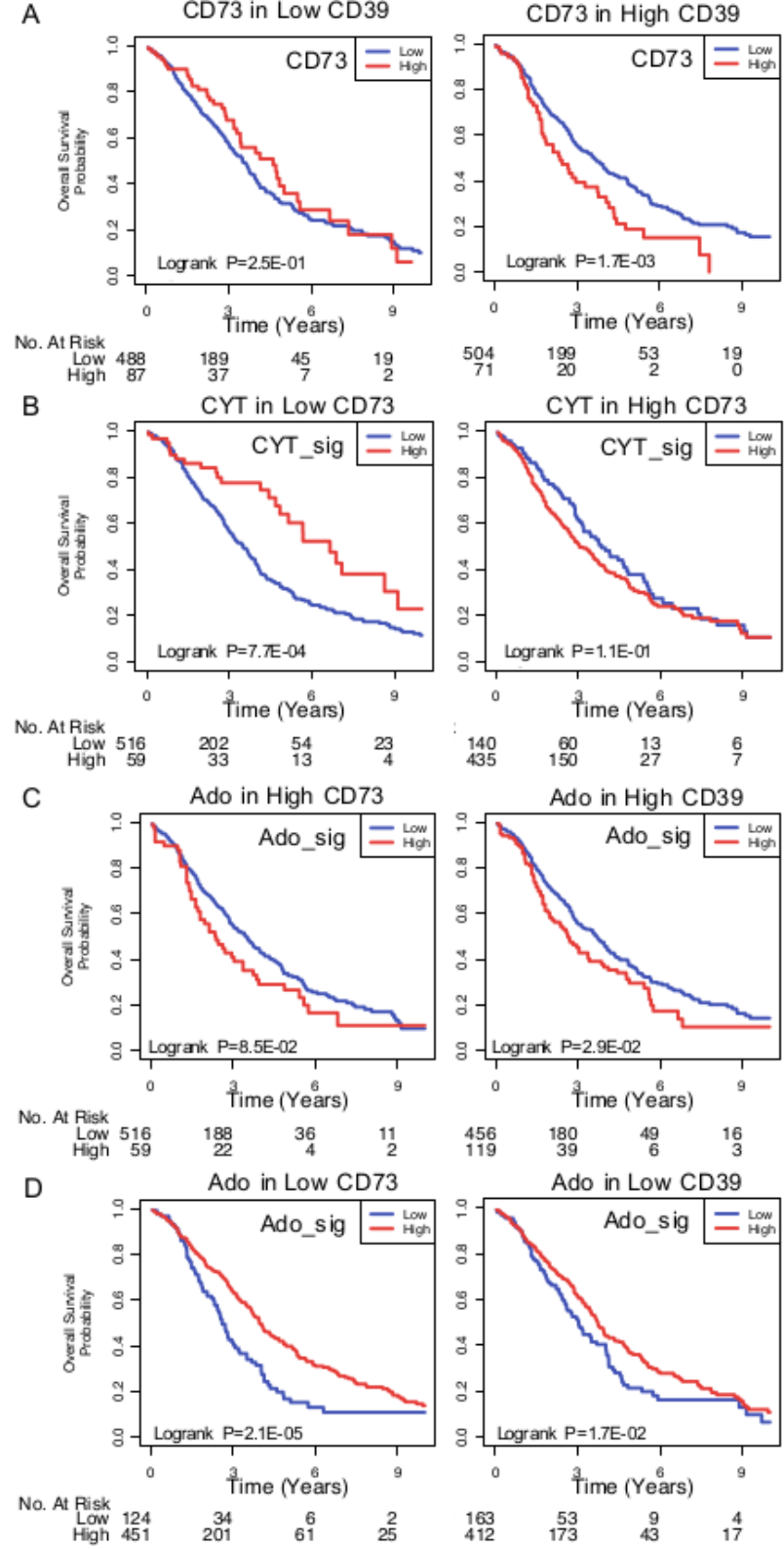

C
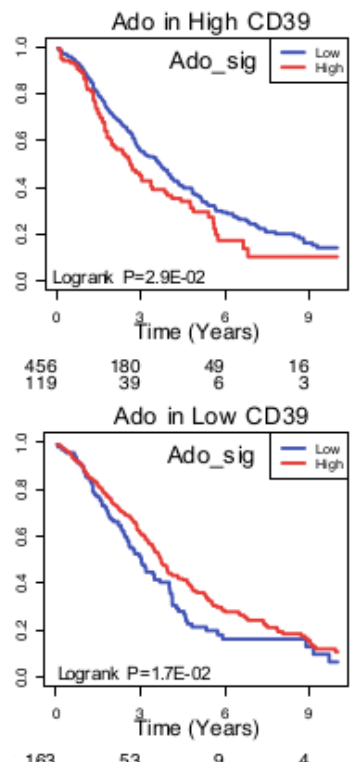

Figure 1 Prognostic impact of the eADO pathway in HGSC ( $\mathrm{n}=1161$ patients). (A) High CD73 (NT5E) expression was associated with worse overall survival in tumors with high CD39 (ENTPD1) expression. (B) Overall survival analysis of high and low immune cytotoxic (CYT) signature according to CD73 gene expression. (C, D) Overall survival analysis of high and low adenosine signature (ADO_sig) according to CD73 or (D) CD39 gene expression. eADO, extracellular adenosine; HGSC, high-grade serous ovarian cancer.

Protein expression was quantified as the mean fluorescence intensity (MFI). Batch effects were removed using COMBAT R package. ${ }^{32}$ Postcombat MFI data were $\log 10$ transformed prior to analysis. Mean expression of duplicate cores was used for analysis. Correlation between duplicates was significant (Spearman's $r>0.6 ; \mathrm{p}<0.001$ ). Cox proportional hazards (coxph) regression was used for univariable and multivariable models ${ }^{19}$ adjusting for age ( $\leq 60$ years vs $>60$ years), stage (I/II vs III/IV) and debulking (optimal vs suboptimal). Where indicated, 
A
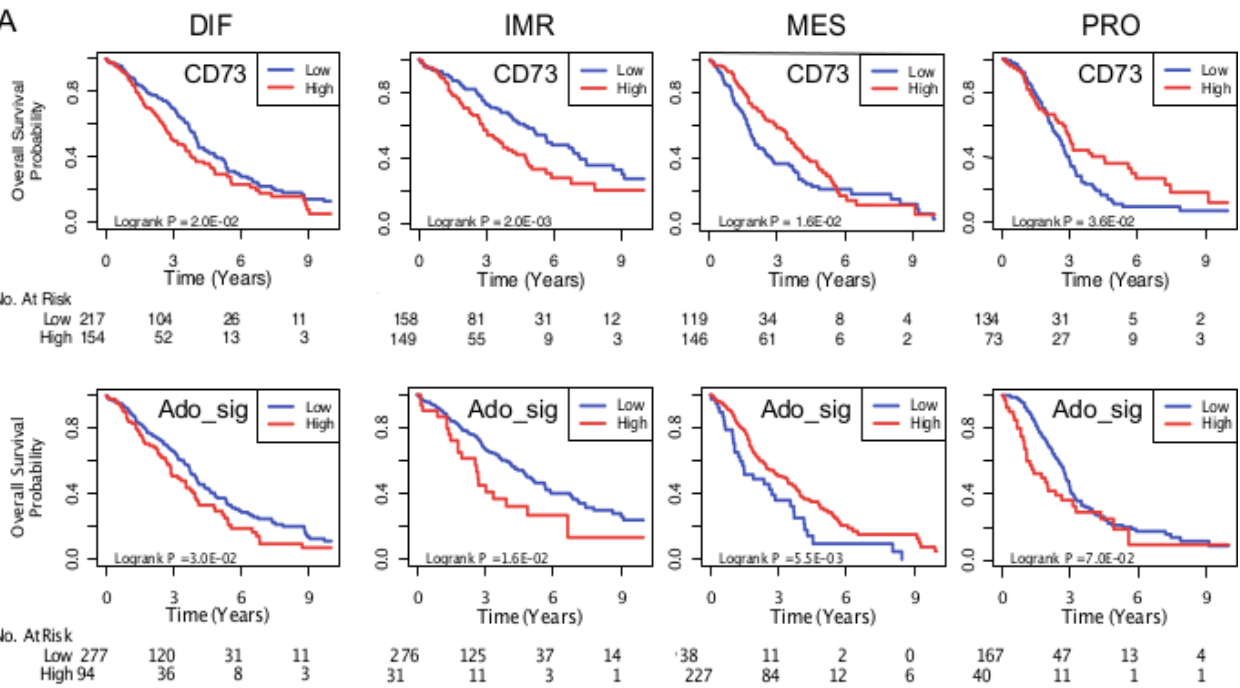

38
227

B

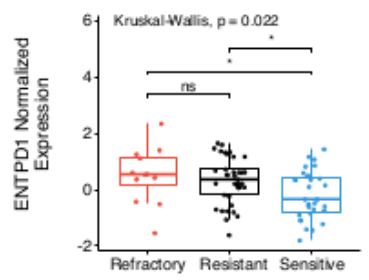

$\mathrm{D}$

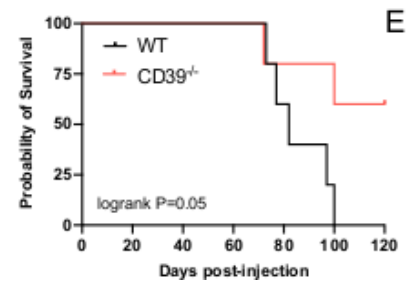

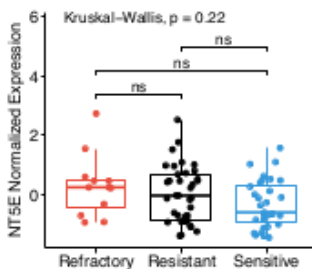

$E$

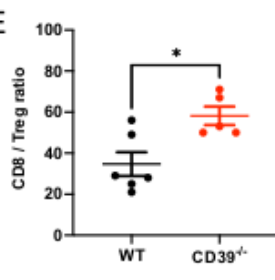

C

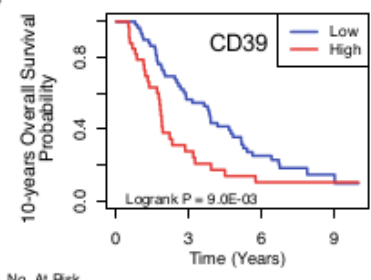

$\begin{array}{rrrrr}\text { No. At Psisk } & & & & \\ \text { Low } & \infty 0 & 30 & 12 & 3 \\ \text { Hgh } & 30 & 8 & 3 & 1\end{array}$

Figure 2 eADO pathway according to molecular subtypes and response to chemotherapy. (A) Subtype-specific prognostic impact of CD73 (NT5E) and ADO_sig in a meta-analysis ( $\mathrm{n}=1161$ patients). HGSC molecular subtypes: differentiated (DIF), immunoregulatory (IMR), mesenchymal (MES) and proliferative (PRO). (B) CD39 (ENTPD1) and CD73 (NT5E) expression was evaluated in pretreatment primary refractory, resistant or sensitive HGSC ( $n=93$ patients). (C) Same as B, overall survival analysis was performed. (D) WT and CD39/- mice were injected i.p. with syngeneic HGS2 mouse ovarian cancer cells ( $10^{7}$ cells) and treated with carboplatin $(20 \mathrm{mg} / \mathrm{kg}$ i.p. Weekly on weeks 5,6 and 7$)$. Overall survival is shown ( $\mathrm{n}=5 / \mathrm{group}$; log RANK $\mathrm{P}$ shown). (E) Tumor-infiltrating $\mathrm{CD} 8^{+} \mathrm{T}$ cells (CD8) and Foxp3 ${ }^{+} \mathrm{CD} 4^{+} \mathrm{T}$ cells (Tregs) of HGS2 tumors were analyzed by flow cytometry at day 12. The ratio of CD8/Tregs in individual tumors, means and SEs are shown ( ${ }^{*} p<0.05$ by Student's t-test). eADO, extracellular adenosine; HGSC, high-grade serous ovarian cancer; ns, not significant; Tregs, T regulatory cell.

protein expression was stratified into two groups based on CK8-18 or aSMA expression. Optimal cut-points were computed for each variable using the surv_cutpoint function form the survminer $\mathrm{R}$ package. Statistical significance of the difference in survival was computed using the Cox proportional hazard model and the log-rank test $p$ value reported on Kaplan-Meier survival curves. All statistical analyses were performed as two sided and, when needed, $\mathrm{p}$ values were adjusted for multi-testing using the Benjamini and Hochberg correction (false discovery rate) using the p.adjust function.

\section{RESULTS}

\section{Prognostic and immunological impacts of eADO signaling in} HGSC

We performed a gene expression meta-analysis of eADO-producing CD39 and CD73, eADO receptor gene signature $^{22}$ and immune gene signatures in 1,161 HGSC patients. CD73 gene expression was significantly associated with worse PFS and OS (online supplemental figure S1A) and its prognostic impact was restricted to tumors with concomitant high levels of CD39 (figure 1A). Consistent with the immunosuppressive effect of eADO, high levels of CD73 abrogated the good prognosis associated with high immune CYT signature (figure 1B), while CD39 expression had no impact (online supplemental figure S1B). An eADO gene signature (ADO_sig) was also associated with worse OS in patients with tumors with high levels of CD73 or CD39 (figure 1C,D). Interestingly, ADO_sig was positively correlated with immune CYT gene signature, IFN gene signature and T cell exhaustion genes (online supplemental figure S2A).

We next investigated whether the eADO pathway was associated with specific molecular subtypes of HGSC. ${ }^{20}$ 
Together, CD39, CD73 and ADO_sign were highest in the immunoregulatory (IMR) and mesenchymal (MES) subtypes, and lowest in the differentiated (DIF) and proliferative (PRO) subtypes (online supplemental figure S2B). CYT score was expectedly highest in the IMR subtype (online supplemental figure S2B). The prognostic impacts of CD73 and ADO_sig were also subtypespecific: they were associated with worse OS in the IMR and DIF subtypes, but associated with better OS in the MES and PRO subtypes (figure 2A).

Since CD39 has been associated with decreased immunogenic cell death triggered by chemotherapy, ${ }^{7}$ we evaluated whether pretreatment CD39 expression was associated with chemoresistance in patients with HGSC. In a characterized cohort of 93 HGSC with primary refractory, resistant or sensitive disease, ${ }^{24}$ we observed that pretreatment CD39 gene expression, but not CD73 expression, was significantly higher in primary refractory and resistant HGSC compared with chemosensitive HGSC (figure 2B), and was associated with worse OS (figure 2C). Interestingly, we observed a significant negative correlation between CD39, CD73 or ADO_sig and $B C R A$ signature, ${ }^{24}$ but no correlation with mismatch repair, APOBEC or AGE signatures (online supplemental figure S3). Since our correlative data suggested a role for CD39 in promoting chemoresistance, we compared the therapeutic activity of carboplatin in a syngeneic mouse model of HGSC implanted into WT and CD39-deficient mice. As shown in figure 2D, chemotherapy with carboplatin was significantly more effective in CD39-deficient mice compared with WT mice, demonstrating a role for host-derived CD39. Notably, host-derived CD39 significantly altered the ovarian tumor immune landscape by decreasing the ratio of infiltrating CD8/Tregs (figure 2E).

\section{Tissue distribution and impact of tumor-derived CD73}

Using quantitative IF, we next assessed what cell types within HGSC tumors expressed CD39 and CD73. Both ectonucleotidases were found to be predominantly expressed by activated $\left(\alpha \mathrm{SMA}^{+}\right)$stromal cells (figure $\left.3 \mathrm{~A}\right)$. CD39 was consistently absent from tumor cells, while high tumorous levels of CD73 were observed in approximately
A

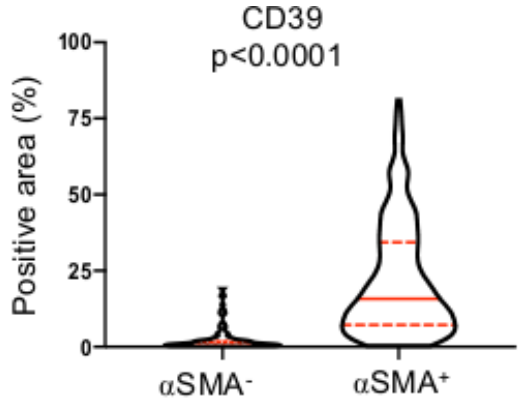

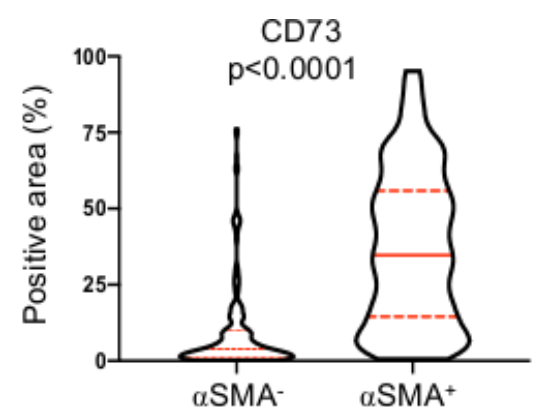

CD39
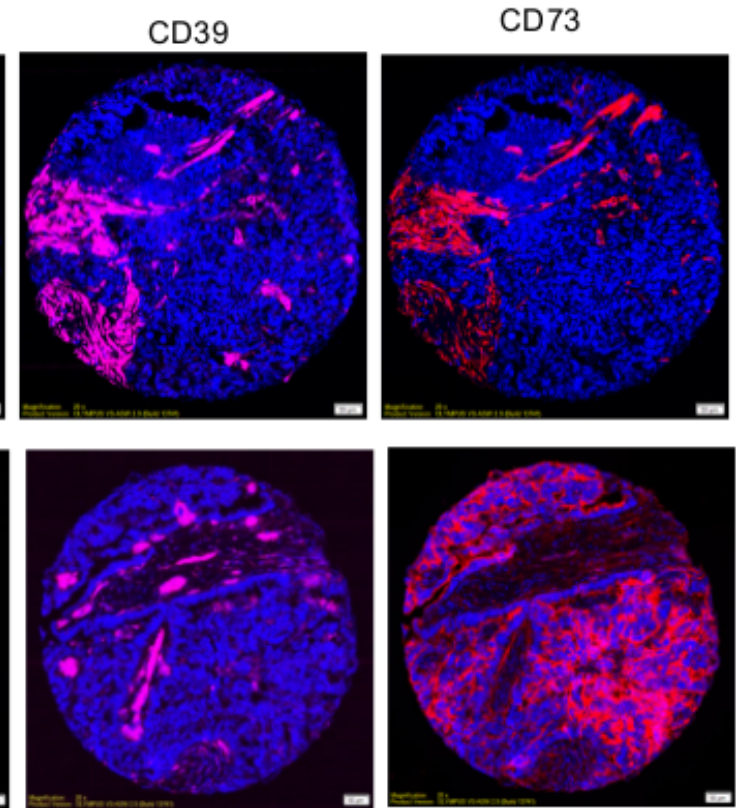

Figure 3 CD39 and CD73 are predominantly expressed in the activated stroma of HGSC. (A) Coimmunofluorescence analysis of $\alpha$ SMA, CD39 and CD73 protein expression in a cohort of 200 HGSC on tissue microarray (median, 95\% Cls and MannWhitney $U$ test $p$ values are shown). (B) Representative staining of a tumor ( $\mathrm{Pt}$ a) with coexpression of CD39 and CD73 in activated $\left(\alpha \mathrm{SMA}^{+}\right)$stroma, and a tumor (Pt B) with high CD73 expression in tumor cells. HGSC, high-grade serous ovarian cancer. 

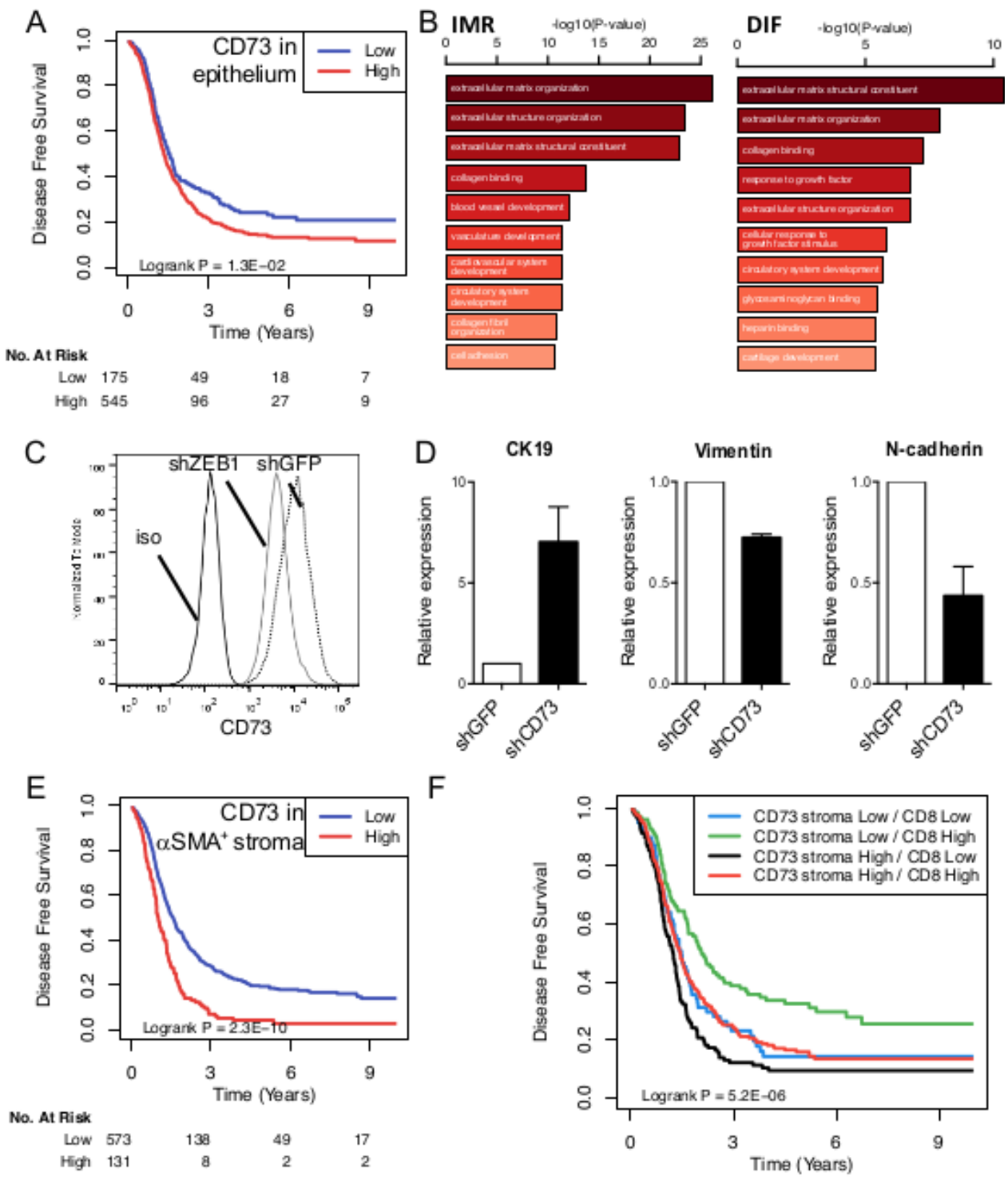

$\mathrm{F}$

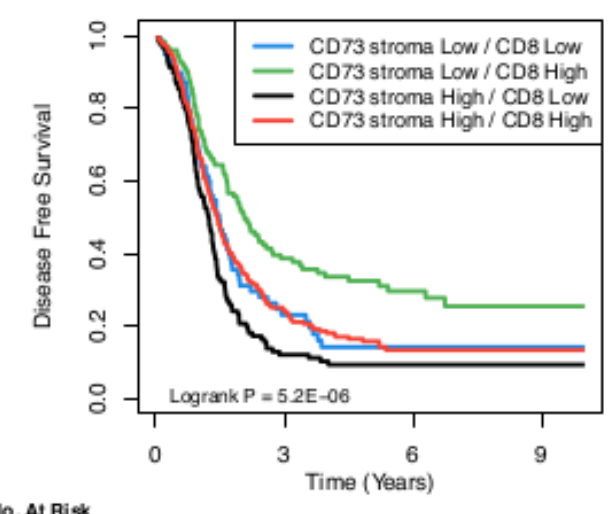

$\begin{array}{lrrrr}\text { CD73 stroma Low / CD8 Low } & 80 & 14 & 6 & 1 \\ \text { CD73 stroma Low / CD8 High } & 128 & 42 & 17 & 6 \\ \text { CD73 stroma High / CD8 Low } & 140 & 14 & 3 & 1 \\ \text { CD73 stroma High / CD8 High } & 237 & 49 & 11 & 5\end{array}$

Figure 4 CD73 protein expression in HGSC is associated with worse prognosis and promotes epithelial-to-mesenchymal transition (EMT). (A) Disease-free survival analysis of CD73 protein expression in epithelial (cytokeratin ${ }^{+}$) areas of HGSC tumors ( $n=720$ patient). (B) Gene ontology analysis of CD73-High immunoregulatory (IMR) and differentiated (DIF) HGSC. (C) Genesilencing of ZEB1 was performed in human SKOV-3 ovarian tumor cells and CD73 surface expression measured by flow cytometry. (D) Gene-silencing of CD73 was performed in SKOV-3 cells and cytokeratin (CK)-19, vimentin and N-cadherin protein expression measured by Western blot (means \pm SEs) of three experiments are shown. (E) Disease-free survival analysis of CD73 protein expression in $\alpha \mathrm{SMA}^{+}$stromal areas of HGSC tumors $(n=704$ patients). ( $F)$ Disease-free survival analysis according to stromal $\left(\alpha \mathrm{SMA}^{+}\right) \mathrm{CD} 73$ expression and intratumoral CD8 expression ( $n=585$ patients). HGSC, high-grade serous ovarian cancer.

$5 \%$ of cases (figure 3B and online supplemental figure S4A). CD73 expression was not associated with age, cytoreductive surgery (ie, debulking) or BRCA mutations, but was higher in advanced stages (online supplemental figure S4B). Survival analysis revealed that higher CD73 expression in tumor cells was significantly associated with worse disease-free survival (figure 4A). To gain insights in the molecular pathways associated with CD73 expression, we performed GO analysis focusing on DIF and IMR molecular subtypes (the two subtypes where CD73 is associated with worse prognosis). CD73-high tumors were enriched for GO pathways promoting extracellular matrix remodeling (figure 4B). Notably, the top correlated genes with $\mathrm{CD} 73$ are known to promote epithelial-tomesenchymal transition (EMT) (online supplemental figure S5). Accordingly, gene silencing of the pro-EMT transcription factor ZEB1 in human in ovarian tumor cells significantly downregulated CD73 surface expression (figure 4C), and CD73 gene silencing significantly upregulated epithelial cytokeratin-19 and downregulated 


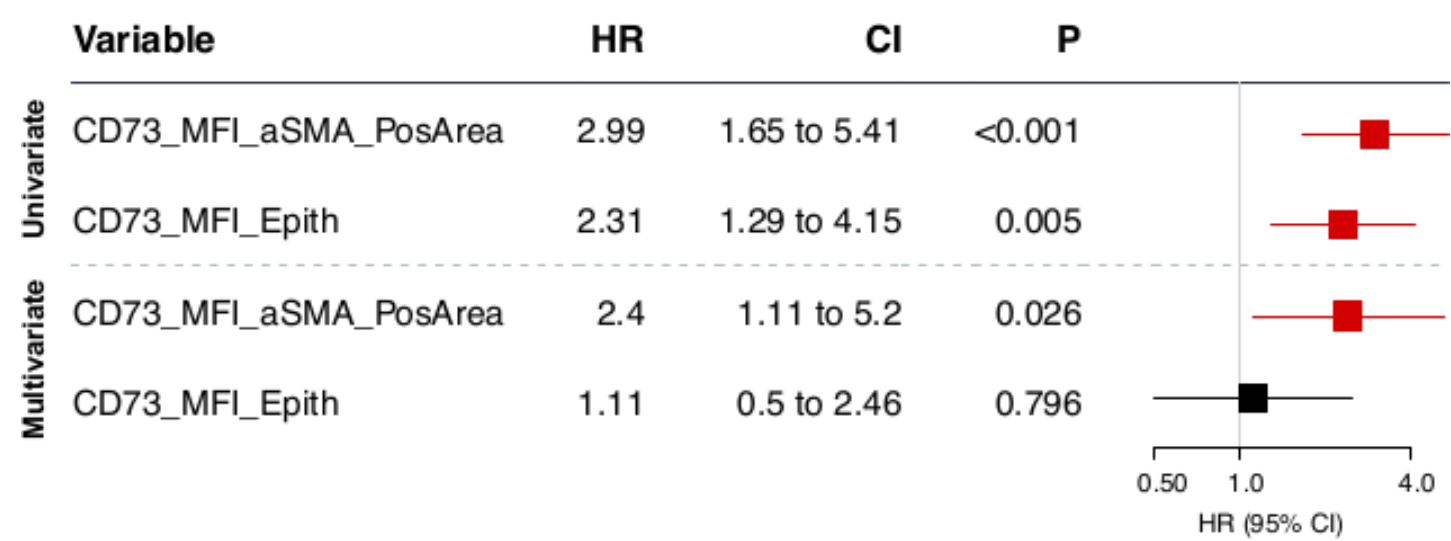

Figure 5 Overall survival univariate and multivariate analysis. Cox proportional hazards models, ${ }^{19}$ adjusting for age, stage and cytoreductive surgery (debulking), for CD73 protein expression (ie, MFI) as a continuous variable within $\alpha \mathrm{SMA}^{+}$stromal area (CD73_MFI_aSMA_PosArea) or epithelial (cytokeratin ${ }^{+}$) areas. MFI, mean fluorescence intensity.

MES vimentin and N-cadherin in human ovarian tumor cells (figure 4D).

\section{Impact of stroma-derived CD73}

We next evaluated the prognostic impact of stromal CD73 expression. High levels of CD73 protein expression by activated $\left(\alpha \mathrm{SMA}^{+}\right)$stromal fibroblasts were significantly associated with worse patient survival (figure 4E). Although we observed a similar trend for stromal CD39 expression, it did reach not statistical significance (online supplemental figure S6). When we evaluated the combined prognostic impact of stromal CD73 and tumorinfiltrating $\mathrm{CD}^{+}$cells, we observed that patients with tumors with high levels of stromal CD73 and low levels of CD8 had the worse prognosis (figure 4F). As a continuous variable, stromal-derived CD73 protein expression was also significantly associated with worse OS in univariate and multivariate analyses, independently of patient age, cytoreductive surgery and cancer stage (figure 5).

\section{CD39 on TILs}

CD39 expression within the epithelium of HGSC tumors showed an expression pattern suggestive of immune infiltrates and was accordingly associated with better prognosis (figure 6A,B). To gain more insights on CD39 expression in TILs, we performed flow cytometry (online supplemental figure S7) and scRNAseq analysis on purified TILs. Unsupervised tSNE analysis revealed predominant CD39 expression in two $\mathrm{CD} 8^{+}$clusters characterized with high levels of PD-1 and TIGIT (figure 6C and online supplemental figure $\mathrm{S} 7$ ). Notably, one $\mathrm{CD} 8^{+} \mathrm{CD} 39^{+}$cluster expressed higher levels of CD25, CD69, CD38, HLADR, LAIR-1, CD244 and IL-7R (CD127). Within CD4 $4^{+}$TILs, CD39 was expressed in putative Tregs $\left(\mathrm{CD} 25^{\text {high }}\right.$ and TIGIT $^{+}$) (online supplemental figure S7) and in CD4 + T cells expressing high levels of PD-1, TIGIT, HLADR and CD69 (figure 6C).

\section{scRNAseq analysis of immune infiltrates}

ScRNAseq analysis (online supplemental figure S8) of CD $45^{+}$immune cells revealed high expression of CD39 in $\mathrm{CD}^{+} \mathrm{T}$ cells, Tregs, myeloid cells, NK cells and B cells infiltrating HGSC (figure 6D). Within CD8 ${ }^{+}$TILs, CD39 was enriched in the CD8.T4 cluster together with $\mathrm{T}$ cell exhaustion genes, activation marker (41BB), CD103 and CXCL13 (figure 6E). Analysis of DEGs in CD8 ${ }^{+}$ TILs revealed a positive correlation between CD39 and CTLA-4, TIM-3, TIGIT, CXCL13, 41BB, SNX9 (involved in T cell costimulation), ${ }^{9}$ MYO1E (involved in lymphocyte motility) ${ }^{33}$ and LAYN (linked to the suppressive function of TILs) ${ }^{34}$ while the top gene negatively correlated with CD39 was GZMK (online supplemental figure S9), involved in anti-PD-1 activity. ${ }^{35}$ We next investigated whether CD39 expression was associated with $\mathrm{T}$ cell expansion and indeed observed that $\mathrm{CD} 39^{+} \mathrm{CD} 8^{+}$TILs (ie, CD8.T4 cluster) displayed highest clonal expansion (figure 6F) and lowest TCR diversity (figure 6G).

\section{CD39 on fibroblasts or T cells suppresses T cell function}

We finally evaluated the functional impact of CD39 expression on cancer-associated fibroblasts (CAFs) and effector $\mathrm{CD}^{+} \mathrm{T}$ cells using primary OVA-specific mouse $\mathrm{CD}^{+} \mathrm{T}$ cells (OT-1 cells) and fibroblasts derived from CD39-deficient mice. OT-1 cells were cocultured with OVA-expressing mouse ovarian tumor cells (ID8) in the presence or absence of syngeneic fibroblasts. IFN- $\gamma$ production was measured as a marker of $\mathrm{T}$ cell activation. As shown in figure $6 \mathrm{H}, \mathrm{CD} 39$ expression on $\mathrm{CD}^{+} \mathrm{T}$ cells (OT-1 cells) or fibroblasts significantly suppressed IFN- $\gamma$ production by OT-1 cells.

\section{DISCUSSION}

Inhibitors of eADO signaling represent an expanding class of immune-stimulating agents with therapeutic activity. However, their potential in gynecological oncology remains largely unknown. eADO accumulates as a result of ATP hydrolysis, mainly by the ectonucleotidases CD39 and CD73. We here demonstrated that both CD39 and CD73 proteins are predominantly expressed by activated CAFs in human HGSC. Notably, CD73 expression in $\alpha \mathrm{SMA}^{+} \mathrm{CAFs}$ was highly prognostic and independent of age, debulking or stage. Given our 

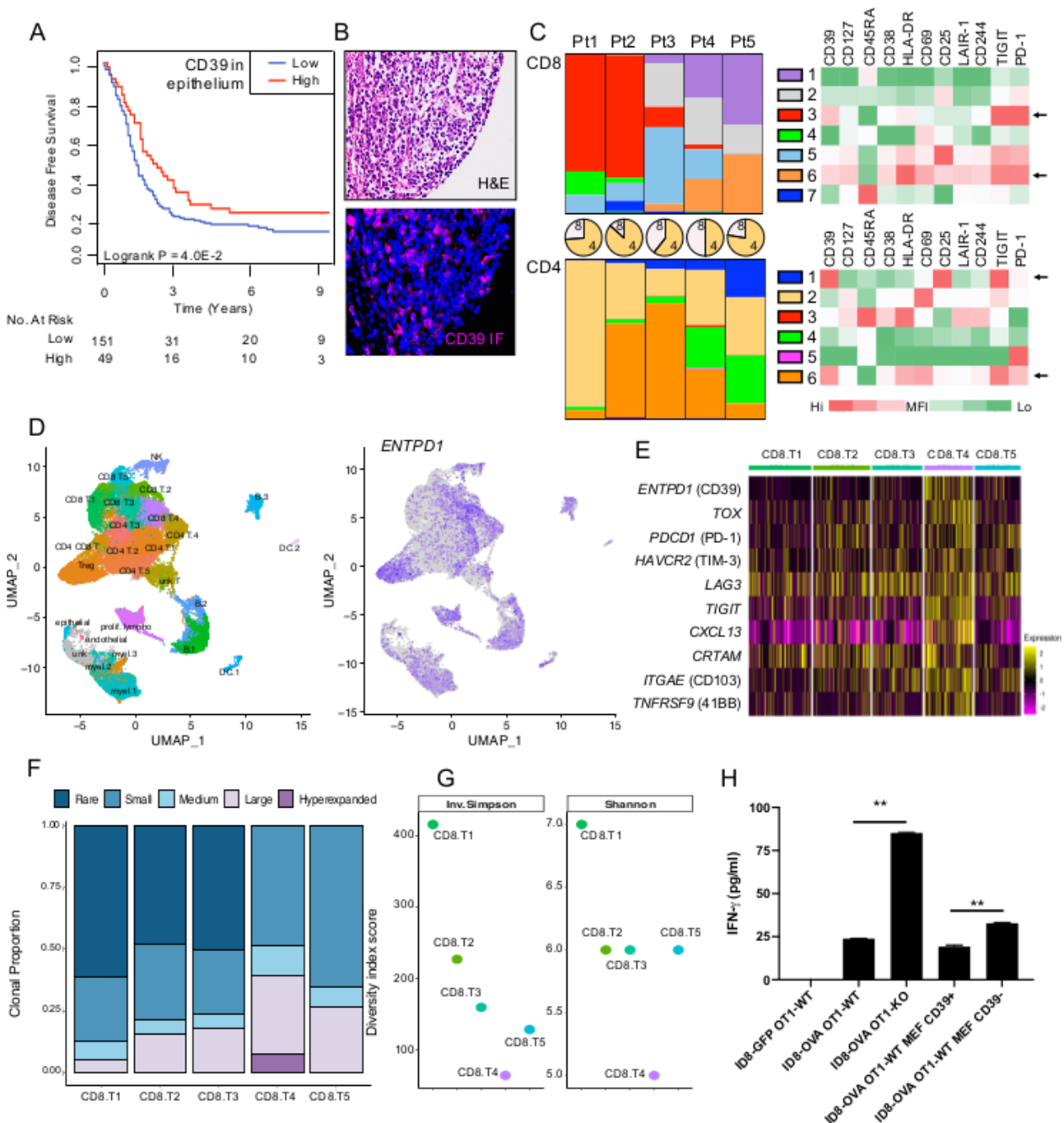

Figure 6 CD39 is expressed by myeloid cells and exhausted clonally expanded tumor-infiltrating T cells. (A) Disease-free survival analysis of high and low CD39 protein expression in epithelium of HGSC tumors ( $n=200$ patient). (B) Representative CD39 staining. (C) Flow cytometry analysis of tumor-infiltrating lymphocytes (TILs) from five HGSC patients. Colored bars represent the proportion of each tSNE cluster within $\mathrm{CD}^{+}$(top) or CD4 ${ }^{+}$TILs (bottom). Pie charts represent the proportion of $\mathrm{CD}^{+}$versus $\mathrm{CD} 4^{+}$TILs. Heatmaps represent relative mean fluorescence intensity (MFI) for each marker within a tSNE cluster. Arrows represent clusters with highest CD39 expression. (D) ScRNAseq analysis of CD45 immune cells isolated from five HGSC patients. UMAP of immune cell clusters (left) and ENTPD1 expression across clusters (right). (E) Expression levels of selected genes in CD8 clusters. (F) Clonal proportion and (G) diversity index of T cell receptors in CD8 clusters were measured. $(\mathrm{H})$ In vitro T cell responses against OVA-expressing ID8 ovarian tumor cells were measured using transgenic mouse OVAspecific CD8 ${ }^{+}$T cells (OT-1) proficient or deficient in CD39 with or without primary mouse embryonic fibroblasts (MEF) proficient or deficient in CD39. IFN- $\gamma$ production was measured by ELISA after 16 hours (means \pm SEs of triplicates are shown; ${ }^{* *} p<0.01$ by Mann-Whitney U test). HGSC, high-grade serous ovarian cancer; OVA, ovalbumin; ScRNAseq, single-cell RNA sequencing;

prior work on CD73-deficient fibroblasts ${ }^{9}$ and our current work on CD39-deficient fibroblasts, our data support a model where CAF-derived ectonucleotidases contribute to ovarian tumor immune escape. High levels of CD73 and CD39 on ovarian CAFs s may, therefore, help stratify patients for eADO-targeting therapy.

In contrast to CAFs, epithelial ovarian tumor cells were only occasionally found to expressed high levels of CD73 (approximately 5\% of cases), while CD39 was consistently absent. Yet, CD39 gene expression in HGSC tumors was significantly associated with chemorefractory and chemoresistant disease. While CD39 may be upregulated following chemotherapy, ${ }^{36}$ our data suggest a prominent role for CD39 expression by 'host' cells, such as CAFs and immune infiltrates in promoting ovarian cancer. In support of this, we observed that CD39-deficient mice with ovarian cancer were significantly more responsive to carboplatin chemotherapy and displayed significantly higher CD8/Tregs ratios in the tumor microenvironment.

Another important finding was that the prognostic impacts of CD73 and ADO_sig were subtype-specific. Accordingly, CD73 and ADO_sig were associated with worse prognosis in the IMR and DIF molecular subtypes of HGSC, but were in contrast associated with better prognosis in the MES and PRO subtypes. Interestingly, CD73 has been previously shown act as a tumor suppressor in 
certain cancers, such as endometrial carcinomas, ${ }^{37}$ and to be associated with better prognosis in ovarian cancers of broad histotype. ${ }^{38}$ Although the clinical significance of molecular classification is still controversial, our data support further investigation of subtype-specific effects eADO signaling in ovarian cancer.

Our study also provides additional support that CD73 is associated with tumor cell stemness. ${ }^{12}$ We indeed demonstrated that EMT was associated with CD73 expression on ovarian tumor cells, and GO analysis revealed a strong correlation between $\mathrm{CD} 73$ and extracellular matrix remodeling and EMT genes. Since EMT has been associated with resistance to immune checkpoint blockade therapy, CD73-mediated EMT may contribute to antiPD-1 treatment resistance in ovarian cancer. ${ }^{39}$

The ADO_sig ${ }^{22}$ here analyzed was found to be associated with worse prognosis in HGSC expressing high levels of CD73 or CD39. Surprisingly, it was associated with better prognosis in tumors expressing low levels of ectonucleotidases. A potential confounding factor may lie in the fact that several genes in the ADO_sig are in fact associated with $\mathrm{T}$ cell activation. Alternatively, high expression of the ADO_sig independently of CD73 or CD39 may reflect alternative eADO-generating pathways. A refinement of the ADO gene signature that better reflects tumor immune landscapes may increase its clinical utility.

Fow cytometry and scRNA-seq analyses revealed that CD39 expression in TILs was highest in exhausted T cells with evidenced of clonal expansion. Our data; thus, support prior studies showing that CD39 identifies exhausted $\mathrm{T}$ cells enriched for tumor antigen recognition. ${ }^{17}{ }^{40}$ Interestingly, a subpopulation of $\mathrm{CD}^{2} 9^{+} \mathrm{CD} 8^{+}$ TILs was found to express CD69, CD127 (IL-7R) and CD103, suggestive of tissue-resident memory $T$ cells $\left(T_{R M}\right.$ cells). Furthermore, coexpression of PD-1 and CD103 in HGSC CD $8^{+}$TILs has been previously shown. ${ }^{41} \mathrm{~A}$ recent study demonstrated a critical role for extracellular ATP and signaling through $\mathrm{P} 2 \times 7$ receptor in the generation of $\mathrm{T}_{\mathrm{RM}}$ cells. ${ }^{42}$ Activation of $\mathrm{P} 2 \times 7$ receptor was shown to controls $\mathrm{T}_{\mathrm{RM}}$ cells by enhancing TGF- $\beta$ signaling. Given that CD39 depletes ATP, CD39 upregulation on T cell exhaustion may, therefore, prevent the development of long-lived $\mathrm{T}_{\mathrm{RM}}$ cells.

Preclinical studies demonstrated that anti-CD39 mAb therapy not only overcomes eADO-mediated immunosuppression, it also increases eATP signaling via $\mathrm{P} 2 \times 7$ receptor leading to increased production of IL- $1 \beta$ and IL-18 in the tumor. ${ }^{43}$ Activation of P $2 \times 7$ and downstream NLRP3 inflammasome are indeed required for antitumor activity of anti-CD39 mAbs. ${ }^{43}$ By engaging the inflammasome pathway, blocking CD39 may thus convert 'immune-cold' tumors into 'immune-active' tumors, which may in turn promote increased sensitivity to chemotherapy, radiotherapy and immune checkpoint blockade.

Selective and potent inhibitors of CD39, CD73, A2A and/or A2B have now entered clinical trials. ${ }^{7}$ Preclinical studies suggest that eADO-targeting agents are most effective when used in combination, for instance with immune checkpoint inhibitors and/or chemotherapy. Combining anti-CD73 mAb with an A2A antagonist also appears synergistic in animal cancer models. ${ }^{44}$ Whether targeting CD39 similarly synergizes with CD73 inhibitors or A2A/ A2B antagonists remains to be investigated. Considering that ovarian cancer patients are currently being treated with eADO inhibitors (NCT03719326, NCT03629756, NCT03454451, NCT03549000) and the therapeutic landscape of HGSC, it would of interest to evaluate combinations of eADO inhibitors with PARP inhibitors, anti-angiogenic agents or emerging immunotherapies. ${ }^{6}$

\section{Author affiliations}

${ }^{1}$ Faculty of Pharmacy, Centre Hospitalier de L'Universite de Montreal, Montreal, Quebec, Canada

${ }^{2}$ Institut du Cancer de Montréal, Centre Hospitalier de L'Universite de Montreal, Montreal, Quebec, Canada

${ }^{3}$ Faculty of Medicine, Departement of Medicine, Centre Hospitalier de L'Universite de Montreal, Montreal, Quebec, Canada

${ }^{4}$ Université Libre de Bruxelles, Institut Jules Bordet, Bruxelles, Bruxelles, Belgium ${ }^{5}$ Divisions of Gastroenterology and Transplantation, Department of Medicine, Beth Israel Deaconess Medical Center, Harvard Med Sch, Boston, Massachusetts, USA

Twitter Mayra Carneiro @carneiro_mayra, Laurence Buisseret @LauBuisseret and Rejean Lapointe @rejeanlapointe

Acknowledgements We thank Dr Yohann Dabi for his constructive feedbacks We thank the patients, the Gynecology-Oncology and Pathology services of all participating centers for tumor procurement. We thank the cytometry core facility of CRCHUM for cell sorting and the molecular pathology core facility of the CRCHUM for performing the HGS2013 and COEUR TMA construction and tissue scanning. Tumor banking for the discovery cohort was supported by the Banque de tissus et de données of the Réseau de recherche sur cancer of the Fonds de recherche Québec - Santé (FRQS) affiliated with the Canadian Tumor Repository Network. This study uses resources provided by the COEUR biobank funded by the Terry Fox Research Institute (TFRI) and managed and supervised by the CHUM. The Consortium acknowledges contributions to its COEUR biobank from Institutions across Canada (www.tfri.ca/COEUR/members).

Contributors YB, SP, MC, LB, IC, PT, PC, LC and DA performed experiments, wrote the manuscript and analyzed data; SCR provided reagents and edited the manuscript; A-MM-M and DP provided reagents and edited the manuscript; RL edited the manuscript; JS analyzed data and wrote the manuscript.

Funding A-MM-M, DP, RL and JS are researchers of CRCHUM, which receive support from the Fonds de recherche Québec - Santé (FRQS). This work was supported by a research grant (Immunotherapy network; iTNT) from the Terry Fox Research Institute to AMMM, RL and JS, by a research grant from the Canadian Institutes of Health Research (CIHR grant FRN 148872) to JS, and by National Institute of Health (NIH) grants (1R21CA221702, R21CA164970 and R01HL094400) to SCR. YB was supported by the Jean-Guy Sabourin Research Chair in Pharmacology of Université de Montréal. LC was supported by a postdoctoral fellowship from FRQS.

Competing interests JS is a SAB member and owns stocks of Surface Oncology. LB received research grants for her Institute from AstraZeneca, speaker honoraria from BMS and travel grant from Roche. SCR received grant support and has served as Consultant to Tizona and is Scientific founder of Purinomia.

\section{Patient consent for publication Not required.}

Ethics approval The study was approved by the Research Ethics Committee of the Montreal University Hospital Center (CHUM) and informed consent was obtained.

Provenance and peer review Not commissioned; externally peer reviewed.

Data availability statement Data are available in a public, open access repository. Data are available in previously reported public repositories.

Supplemental material This content has been supplied by the author(s). It has not been vetted by BMJ Publishing Group Limited (BMJ) and may not have been peer-reviewed. Any opinions or recommendations discussed are solely those of the author(s) and are not endorsed by BMJ. BMJ disclaims all liability and responsibility arising from any reliance placed on the content. Where the content 
includes any translated material, BMJ does not warrant the accuracy and reliability of the translations (including but not limited to local regulations, clinical guidelines, terminology, drug names and drug dosages), and is not responsible for any error and/or omissions arising from translation and adaptation or otherwise.

Open access This is an open access article distributed in accordance with the Creative Commons Attribution Non Commercial (CC BY-NC 4.0) license, which permits others to distribute, remix, adapt, build upon this work non-commercially, and license their derivative works on different terms, provided the original work is properly cited, appropriate credit is given, any changes made indicated, and the use is non-commercial. See http://creativecommons.org/licenses/by-nc/4.0/.

\section{ORCID iDs}

Mayra Carneiro http://orcid.org/0000-0002-5077-1730

John Stagg http://orcid.org/0000-0001-7833-4228

\section{REFERENCES}

1 Hwang W-T, Adams SF, Tahirovic E, et al. Prognostic significance of tumor-infiltrating T cells in ovarian cancer: a meta-analysis. Gynecol Oncol 2012;124:192-8.

2 Zhang L, Conejo-Garcia JR, Katsaros D. Intratumoral T cells, recurrence, and survival in epithelial ovarian cancer. N Engl $J$ Med 2003.

3 Goode EL, Block MS, Kalli KR. Dose-Response association of CD8+ tumor-infiltrating lymphocytes and survival time in high-grade serous ovarian cancer. JAMA Oncol 2017.

4 Matulonis UA, Shapira-Frommer R, Santin AD, et al. Antitumor activity and safety of pembrolizumab in patients with advanced recurrent ovarian cancer: results from the phase II KEYNOTE-100 study. Ann Oncol 2019;30:1080-7.

5 Konstantinopoulos PA, Waggoner S, Vidal GA, et al. Single-Arm phases 1 and 2 trial of Niraparib in combination with pembrolizumab in patients with recurrent platinum-resistant ovarian carcinoma. JAMA Oncol 2019;5:1141.

6 Kandalaft LE, Odunsi K, Coukos G. Immunotherapy in ovarian cancer: are we there yet? J Clin Oncol 2019;37:2460-71.

7 Allard B, Allard D, Buisseret L. The adenosine pathway in immunooncology. Nat Rev Clin Oncol 2020.

8 Allard B, Pommey S, Smyth MJ. Targeting CD73 enhances the antitumor activity of anti-PD-1 and anti-CTLA-4 mAbs. Clin Cancer Res 2013.

9 Turcotte M, Spring K, Pommey S, et al. Cd73 is associated with poor prognosis in high-grade serous ovarian cancer. Cancer Res 2015;75:4494-503.

10 Zhang B. Cd73: a novel target for cancer immunotherapy. Cancer Res 2010;70:6407-11.

11 Wang L, Fan J, Thompson LF, et al. Cd73 has distinct roles in nonhematopoietic and hematopoietic cells to promote tumor growth in mice. J Clin Invest 2011;121:2371-82.

12 Lupia M, Angiolini F, Bertalot G, et al. Cd73 regulates stemness and epithelial-mesenchymal transition in ovarian Cancer-Initiating cells. Stem Cell Reports 2018;10:1412-25.

13 Nevedomskaya E, Perryman R, Solanki S, et al. A systems oncology approach identifies NT5E as a key metabolic regulator in tumor cells and modulator of platinum sensitivity. $J$ Proteome Res 2016:15:280-90.

14 Montalbán Del Barrio I, Penski C, Schlahsa L, et al. Adenosinegenerating ovarian cancer cells attract myeloid cells which differentiate into adenosine-generating tumor associated macrophages - a self-amplifying, CD39- and CD73-dependent mechanism for tumor immune escape. J Immunother Cancer 2016;4:49

15 d'Almeida SM, Kauffenstein G, Roy C, et al. The ecto-ATPDase CD39 is involved in the acquisition of the immunoregulatory phenotype by M-CSF-macrophages and ovarian cancer tumorassociated macrophages: regulatory role of IL-27. Oncoimmunology 2016:5:e1178025.

16 Vuerich M, Harshe R, Frank LA, et al. Altered aryl-hydrocarbonreceptor signalling affects regulatory and effector cell immunity in autoimmune hepatitis. J Hepatol 2021;74:48-57.

17 Duhen T, Duhen R, Montler R, et al. Co-Expression of CD39 and CD103 identifies tumor-reactive CD8 T cells in human solid tumors. Nat Commun 2018;9.

18 Gendoo DMA, Zon M, Sandhu V, et al. MetaGxData: clinically annotated breast, ovarian and pancreatic cancer datasets and their use in generating a Multi-Cancer gene signature. Sci Rep 2019;9.
19 Gendoo DMA, Ratanasirigulchai N, Schröder MS, et al. Genefu: an $\mathrm{R} /$ Bioconductor package for computation of gene expression-based signatures in breast cancer. Bioinformatics 2016;32:1097-9.

20 Chen GM, Kannan L, Geistlinger L, et al. Consensus on molecular subtypes of high-grade serous ovarian carcinoma. Clin Cancer Res 2018;24:5037-47.

21 Wei JS, Kuznetsov IB, Zhang S, et al. Clinically Relevant Cytotoxic Immune Cell Signatures and Clonal Expansion of T-Cell Receptors in High-Risk MYCN-Not-Amplified Human Neuroblastoma. Clin Cancer Res 2018;24:5673-84.

22 Sidders B, Zhang P, Goodwin K, et al. Adenosine signaling is prognostic for cancer outcome and has predictive utility for immunotherapeutic response. Clin Cancer Res 2020;26:2176-87.

23 Färkkilä A, Gulhan DC, Casado J. Immunogenomic profiling determines responses to combined PARP and PD-1 inhibition in ovarian cancer. Nat Commun 2020.

24 Patch A-M, Christie EL, Etemadmoghadam D, et al. WholeGenome characterization of chemoresistant ovarian cancer. Nature 2015;521:489-94.

25 Le Page C, Rahimi K, Köbel M, et al. Characteristics and outcome of the coeur Canadian validation cohort for ovarian cancer biomarkers. BMC Cancer 2018;18.

26 McShane LM, Altman DG, Sauerbrei W, et al. Reporting recommendations for tumor marker prognostic studies (REMARK). Breast Cancer Res Treat 2006;100:229-35.

27 Stuart T, Butler A, Hoffman P, et al. Comprehensive integration of single-cell data. Cell 2019;177:1888-902.

28 Finak G, McDavid A, Yajima M, et al. Mast: a flexible statistical framework for assessing transcriptional changes and characterizing heterogeneity in single-cell RNA sequencing data. Genome Biol 2015;16.

29 Brunner PM, Jonak C, Knobler R. Recent advances in understanding and managing cutaneous T-cell lymphomas. F1000Res 2020;9:331.

30 Maniati E, Berlato C, Gopinathan G, et al. Mouse ovarian cancer models recapitulate the human tumor microenvironment and patient response to treatment. Cell Rep 2020;30:525-40.

31 Ritchie ME, Phipson B, Wu D, et al. limma powers differential expression analyses for RNA-sequencing and microarray studies. Nucleic Acids Res 2015;43:e47.

32 Kothari S, Phan JH, Stokes TH, et al. Removing batch effects from histopathological images for enhanced cancer diagnosis. IEEE J Biomed Health Inform 2014:18:765-72.

33 Girón-Pérez DA, Vadillo E, Schnoor M, et al. Myo1E modulates the recruitment of activated B cells to inguinal lymph nodes. J Cell Sci 2020;133. doi:10.1242/jcs.235275. [Epub ahead of print: 02 Mar 2020]

34 Zheng C, Zheng L, Yoo J-K, et al. Landscape of infiltrating T cells in liver cancer revealed by single-cell sequencing. Cell 2017;169:1342-56.

35 Naing A, Infante J, Goel S, et al. Anti-Pd-1 monoclonal antibody MEDI0680 in a phase I study of patients with advanced solid malignancies. J Immunother Cancer 2019;7.

36 Loi S, Pommey S, Haibe-Kains B, et al. Cd73 promotes anthracycline resistance and poor prognosis in triple negative breast cancer. Proc Natl Acad Sci U S A 2013;110:11091-6.

37 Bowser JL, Blackburn MR, Shipley GL, et al. Loss of CD73-mediated actin polymerization promotes endometrial tumor progression. J Clin Invest 2016;126:220-38.

$38 \mathrm{Oh} \mathrm{HK}$, Sin J-I, Choi J, et al. Overexpression of CD73 in epithelial ovarian carcinoma is associated with better prognosis, lower stage, better differentiation and lower regulatory $T$ cell infiltration. $J$ Gynecol Oncol 2012;23:274-81.

39 Chakravarthy A, Khan L, Bensler NP, et al. TGF- $\beta$-associated extracellular matrix genes link cancer-associated fibroblasts to immune evasion and immunotherapy failure. Nat Commun 2018;9.

40 Simoni $\mathrm{Y}$, Becht E, Fehlings $\mathrm{M}$, et al. Bystander $\mathrm{CD} 8^{+} \mathrm{T}$ cells are abundant and phenotypically distinct in human tumour infiltrates. Nature 2018;557:575-9.

41 Webb JR, Milne K, Nelson BH. Pd-1 and CD103 are widely coexpressed on prognostically favorable intraepithelial CD8 T cells in human ovarian cancer. Cancer Immunol Res 2015;3:926-35.

42 Borges da Silva $\mathrm{H}$, Peng C, Wang $\mathrm{H}$, et al. Sensing of ATP via the Purinergic Receptor P2RX7 Promotes CD8 ${ }^{+}$Trm Cell Generation by Enhancing Their Sensitivity to the Cytokine TGF- $\beta$. Immunity 2020;53:158-71.

43 XY L, Moesta AK, Xiao C. Targeting CD39 in cancer reveals an extracellular ATP-and inflammasome-driven tumor immunity. Cancer Discov 2019.

44 Young A, Ngiow SF, Barkauskas DS, et al. Co-Inhibition of CD73 and A2aR adenosine signaling improves anti-tumor immune responses. Cancer Cell 2016;30:391-403. 